\title{
RELAÇÃO CINEMÁTICA EM UM TRATOR 4x2 COM TRAÇÃO DIANTEIRA AUXILIAR EQUIPADO COM PNEUS RADIAIS NA EFICIÊNCIA DE TRAÇÃOO ${ }^{1}$
}

\author{
KINEMATIC RELATION ON RADIAL TIRES IN A FRONT WHEEL ASSIST TRACTOR \\ ON TRACTION EFFICIENCE
}

\author{
Mauro Fernando Ferreira ${ }^{2}$ José Fernando Schlosser ${ }^{3}$ Eduardo Briese Neujahr ${ }^{4}$ \\ Virgílio Schneider ${ }^{4}$
}

\section{RESUMO}

Diferentes combinações de pressões internas dos pneus do trator pode afetar a interferência entre eixos motrizes dos tratores agrícolas, principalmente com pneus do tipo radial. Um trator $4 x 2$ com tração dianteira auxiliar foi analisado quanto a seu desempenho em tração. Pneus de carcaça radial com diferentes pressões internas foram utilizados, com o objetivo de variar as relações cinemáticas entre os eixos. Mediram-se o patinamento das rodas dianteiras e traseiras, a resistência ao rolamento e a força de tração, em duas condições de solo (firme e solto). Os resultados obtidos permitiram verificar que a eficiência de tração não foi significativamente influenciada pela variação das relações cinemáticas de 0,962 a 1,102. As máximas eficiências de tração ocorreram com relações cinemáticas variávei dentro da faixa estudada e de acordo com as cargas impostas à barra de tração.

Palavras-chave: pneus agrícolas, tração, patinamento.

\section{SUMMARY}

Different combinations of tractor tire inflating pressure may affect interference between tractor axles, mainly with radial tires type. A front wheel assist tractor was studied in its traction performance. Radial tires with different inflation pressure were used, changing kinematic relations between axles. The measured parameters were: front and rear slip, rolling resistence and drawbar pull in two soil conditions (firm and loose). The results indicate that traction efficience was not significantly influenced by kinematic relations variation between 0.962 to 1.102. The maximum traction efficiency ocurred within the range studied and according to drawbar pull.

Key words: agricultural tires, traction, slip.

\section{INTRODUÇÃO}

O trator agrícola é uma unidade polivalente, que realiza diversos trabalhos e em condições muito variadas (MÁRQUEZ, 1990). Essa máquina constitui-se, segundo SCHLOSSER (1997), na principal fonte de potência para a agricultura, servindo de base à mecanização agrícola moderna. Cerca de $60 \%$ dos modelos de tratores, atualmente, disponíveis apresentam tração dianteira auxiliar (TDA), existindo uma versão TDA para cada modelo de tração com duas rodas motrizes (2RM), o que demonstra a grande aceitação no mercado nacional (YANAI $\boldsymbol{e t} \boldsymbol{a l} ., 1998)$. Essa aceitação pode ser atribuída, segundo CORRÊA et al. (1998), à capacidade superior de tração desses tratores, principalmente em condições de solo mais adversas.

Os tratores agrícolas com tração dianteira auxiliar possuem uma ligação rígida entre os eixos dianteiros e traseiros, quando o operador aciona a TDA. Com o sistema conectado, o eixo dianteiro gira com uma rotação superior ao eixo traseiro, denominada adianto cinemático. Esse adianto varia de modelo para modelo de trator de acordo com critérios do fabricante e tem a função de corrigir as diferenças de diâmetros entre as rodas do eixo dianteiro e traseiro. Como as velocidades periféricas das duas

\footnotetext{
${ }^{1}$ Parte da dissertação de Mestrado do primeiro autor.

${ }^{2}$ Engenheiro Agrícola, Mestrando do Programa de Pós-graduação em Engenharia Agrícola, Departamento de Engenharia Rural (DER), Centro de Ciências Rurais (CCR), Universidade Federal de Santa Maria (UFSM), 97105-900, Santa Maria, RS.

${ }^{3}$ Professor Titular, DER, CCR, UFSM. E-mail: schlosse @ ccr.ufsm.br. Autor para correspondência.

${ }^{4}$ Engenheiro Agrônomo, Mestrando do Programa de Pós-graduação em Engenharia Agrícola DER, CCR, UFSM. 
rodas devem ser aproximadamente iguais, os fabricantes recomendam pares compatíveis de pneus, deixando essas velocidades aproximadamente iguais (LINARES, 1996). Segundo SCHLOSSER (1996), pesquisas demonstram que com a utilização desses pneus recomendados, a rotação do eixo dianteiro deve ser um pouco superior à rotação do eixo traseiro, com a ligação entre os eixos rígida, sistema este denominado de relação cinemática $(\mathrm{Kv})$. Esta relação pode ser alterada, já que depende da pressão interna dos pneus, carga sobre os pneus, transferência de peso e do desgaste diferenciado dos pneus (RACKHAM \& BLIGHT, 1985).

Este trabalho teve como objetivo principal a comparação do desempenho da eficiência de tração de um trator com TDA, com diferentes relações cinemáticas, em duas condições de solo, utilizando um trator $4 \times 2$ com TDA, equipado com pneus radiais.

\section{MATERIAL E MÉTODOS}

O trabalho foi realizado no Campus Universitário da Universidade Federal de Santa Maria. O experimento foi conduzido em duas condições de solo, separadas em duas parcelas planas e adjacentes. A primeira condição consistia em um solo solto, preparado mecanicamente com operação de aração com arado de discos a uma profundidade de $20 \mathrm{~cm}$ e seguido de duas gradagens com grade niveladora de discos. A segunda condição consistia em um solo firme, sem preparo prévio, com uma cobertura de aveia roçada momentos antes da realização dos testes. As avaliações de resistência à penetração realizadas com um penetrômetro, marca Soilcontrol, modelo SC-60, apresentaram valores de índice de cone de $2.041 \mathrm{kPa}$ para o solo firme, enquanto que para o solo solto o índice de cone era $449 \mathrm{kPa}$, até a profundidade de $15 \mathrm{~cm}$. O conteúdo gravimétrico de água do solo solto estava em $18 \%$ e no solo firme em $16,2 \%$

O trator avaliado foi da marca Massey Ferguson, modelo 620, com potência bruta no motor de $76,92 \mathrm{~kW}$, turboalimentado, cilindrada de $4100 \mathrm{~cm}^{3}$ e com TDA. A massa total desse trator era de $5.406 \mathrm{~kg}$, com uma distribuição estática de peso de $2.256 \mathrm{~kg}$ dianteiro e $3.150 \mathrm{~kg}$ traseiro $(41,73 \%$ dianteiro - 58,27\% traseiro). Para os testes, não foi colocado nenhum tipo de lastro líquido, mas o trator estava lastrado com 8 contrapesos metálicos dianteiros de $45 \mathrm{~kg}$. O trator de testes, durante as avaliações, foi colocado em 3. ${ }^{a}$ marcha (Reduzida/Baixa), com uma rotação no motor de 1800 rotações. min $^{-1}$, permanecendo com o bloqueio do diferencial e a TDA ligados. O adianto cinemático foi determinado, ficando em 1,336. Os pneus radiais utilizados foram da marca MICHELIN modelo XM-27, com banda de rodagem R1 e designação técnica 480/65R24 127
A8 para os dianteiros e 620/75R26 166 A8 para os traseiros. Para criar uma resistência e conseqüentemente uma carga, necessária para o experimento com dinamometria, foi utilizado um trator de freio da marca Ford, modelo 7610, com TDA. As pressões internas dos pneus mudaram conforme os tratamentos, para se obterem diferentes relações cinemáticas. As pressões internas dos pneus dianteiros e traseiros alteraram significativamente os raios dinâmicos dos pneus variando da relação cinemática (Tabela 1). As alterações das pressões foram realizadas no sentido das maiores para as menores, de modo que fosse utilizado o mínimo possível o compressor de ar, facilitando o trabalho de campo.

$\mathrm{O}$ trator de teste foi instrumentado com uma plataforma dotada de instrumentação constituída por um conjunto dinamométrico da marca Alfa Instrumentos, para a determinação da força de tração, através de uma célula de carga de capacidade de $50 \mathrm{kN}$, colocada entre os tratores. Para unir os dois tratores, passando pela célula de carga, foi utilizado um cabo de aço. $\mathrm{O}$ patinamento das rodas motrizes foi determinado pelo método das balizas, medindose a distância percorrida pelas rodas em cinco voltas. As marcações foram feitas com o auxílio de oito balizas, quatro para as rodas dianteiras e quatro para as rodas traseiras. Nos pneus do trator, foram feitas marcações próximas às válvulas para identificar o ponto onde a volta deveria ser contada. A seguir, com o auxílio de uma trena de 50 metros, foi medida a distância percorrida pelas rodas, para o posterior cálculo do patinamento em função da distância percorrida no mesmo número de voltas em uma superfície de referência, no caso, o solo firme. Foi determinada também a força de tração exercida pelo trator de teste, variando três cargas no trator de freio em cada relação cinemática, bem como a medição da resistência ao rolamento em todas as relações cinemáticas.

Foram utilizados quarenta e oito tratamentos decorrentes da combinação entre oito rela-

Tabela 1 - Pressões internas, raios estáticos e relações cinemáticas para o trator MF 620 com pneus radiais.

\begin{tabular}{ccccc}
\hline \multicolumn{2}{c}{ Pneu dianteiro } & \multicolumn{2}{c}{ Pneu traseiro } & \\
\cline { 1 - 3 } $\begin{array}{l}\text { Pressão } \\
\text { interna } \\
(\mathrm{kPa})\end{array}$ & $\begin{array}{c}\text { Raio estático } \\
(\mathrm{mm})\end{array}$ & $\begin{array}{c}\text { Pressão } \\
\text { interna } \\
(\mathrm{kPa})\end{array}$ & $\begin{array}{c}\text { Raio } \\
\text { estático } \\
(\mathrm{mm})\end{array}$ & $\begin{array}{c}\text { Relação } \\
\text { cinemática } \\
(\mathrm{Kv})\end{array}$ \\
\cline { 1 - 2 } & & & & \\
\hline & & & & \\
172,4 & 585 & 62,0 & 709 & 1,102 \\
172,4 & 585 & 89,6 & 729 & 1,072 \\
172,4 & 585 & 158,6 & 751 & 1,041 \\
117,2 & 567 & 158,6 & 751 & 1,009 \\
89,6 & 554 & 158,6 & 751 & 0,986 \\
75,8 & 541 & 158,6 & 751 & 0,962 \\
& & & &
\end{tabular}


ções cinemáticas (seis para os pneus radiais e duas para diagonais), duas condições de solo (firme e solto) e três níveis de tração na barra. Para cada um dos tratamentos, foram utilizadas três repetições. A carga imposta ao trator de ensaio foi definida pela variação de resistência criada pelo trator freio, variando em função da condição de solo, de maneira a obter patinamentos dentro de uma faixa entre os $10 \mathrm{e}$ $40 \%$.

A análise estatística foi realizada através do pacote estatístico SAS, utilizando um delineamento de blocos ao acaso, em fatorial, determinando o coeficiente de variação entre os valores medidos e calculados, análise de variância e um teste de diferenças de médias.

\section{RESULTADOS E DISCUSSÃO}

Os resultados dos testes de campo, com relação aos patinamentos traseiros, dianteiros, médio e eficiência de tração, estão apresentados nas tabelas 2 e 3. Na tabela 2, observa-se que os patinamentos das rodas traseiras em solo firme variaram significativamente em função da relação cinemática $(\mathrm{kv})$. Os patinamentos dianteiros e patinamentos médios entre os dois eixos não foram significativamente diferentes, em função das relações cinemáticas, ou seja, a variação das relações cinemáticas não influenciaram esses patinamentos. Os patinamentos dianteiros foram sempre maiores do que os patinamentos traseiros, mesmo com relações cinemáticas de atraso.

Quanto à eficiência de tração no solo firme, observa-se pela tabela 2 que não houve diferença entre as eficiências de tração, em função da variação das relações cinemáticas entre os eixos.

$\mathrm{Na}$ figura 1, é apresentada a influência da relação cinemática na eficiência de tração em função das cargas impostas à barra de tração. Observa-se que quanto menor a carga imposta à barra de tração, maior é a eficiência de tração, independentemente da relação cinemática usada. Na carga 1 e na carga 2 , a eficiência mostra a tendência de estabilização e indica que as maiores eficiências de tração estão na faixa de relações cinemáticas entre 0,986 e 1,041, embora na relação 1,009 observe-se uma pequena queda na eficiência de tração. Para a carga 3, observa-se que ocorre uma estabilização das eficiências na faixa de relações entre 0,962 e 1,041 , com uma pequena queda da eficiência na relação 1,009. Para relações maiores do que 1,041 , nota-se uma pequena, porém significativa, tendência do aumento da eficiência de tração, ficando nessa faixa as maiores eficiências de tração.

Os patinamentos das rodas traseiras em solo solto variaram significativamente em função da relação cinemática $(\mathrm{kv})$. Os patinamentos dianteiros
Tabela 2 - Patinamento dos pneus radiais traseiros, dianteiros, médio e eficiência de tração em função do solo firme, relação cinemática e cargas na barra de tração.

\begin{tabular}{|c|c|c|c|c|c|}
\hline \multirow{2}{*}{$\begin{array}{c}\text { Relação } \\
\text { Cinemática } \\
\text { (Kv) }\end{array}$} & \multirow[b]{2}{*}{ Carga } & \multicolumn{3}{|c|}{ Patinamentos } & \multirow{2}{*}{$\begin{array}{c}\text { Eficiência } \\
\text { de Tração } \\
(\%)\end{array}$} \\
\hline & & $\begin{array}{c}\text { Traseiro } \\
(\%)\end{array}$ & $\begin{array}{c}\text { Dianteiro } \\
(\%)\end{array}$ & $\begin{array}{c}\text { Médio } \\
(\%)\end{array}$ & \\
\hline 0,962 & 1 & $11,36^{\mathrm{ab}}$ & $16,67^{\mathrm{a}}$ & $14,02^{\mathrm{a}}$ & $0,659^{\mathrm{a}}$ \\
\hline 0,962 & 2 & $19,99^{\mathrm{ab}}$ & $23,22^{\mathrm{a}}$ & $21,61^{\mathrm{a}}$ & $0,609^{\mathrm{a}}$ \\
\hline 0,962 & 3 & $35,50^{\mathrm{ab}}$ & $39,72^{\mathrm{a}}$ & $37,61^{\mathrm{a}}$ & $0,491^{\mathrm{a}}$ \\
\hline 0,986 & 1 & $10,58^{\mathrm{a}}$ & $16,39^{\mathrm{a}}$ & $13,49^{\mathrm{a}}$ & $0,656^{\mathrm{a}}$ \\
\hline 0,986 & 2 & $19,74^{\mathrm{a}}$ & $24,63^{\mathrm{a}}$ & $22,19^{\mathrm{a}}$ & $0,599^{\mathrm{a}}$ \\
\hline 0,986 & 3 & $35,39^{\mathrm{a}}$ & $40,34^{\mathrm{a}}$ & $37,87^{\mathrm{a}}$ & $0,486^{\mathrm{a}}$ \\
\hline 1,009 & 1 & $11,71^{\mathrm{a}}$ & $17,86^{\mathrm{a}}$ & $14,79^{\mathrm{a}}$ & $0,639^{\mathrm{a}}$ \\
\hline 1,009 & 2 & $18,70^{\mathrm{a}}$ & $24,12^{\mathrm{a}}$ & $21,41^{\mathrm{a}}$ & $0,594^{\mathrm{a}}$ \\
\hline 1,009 & 3 & $38,48^{\mathrm{a}}$ & $41,83^{\mathrm{a}}$ & $40,16^{\mathrm{a}}$ & $0,464^{\mathrm{a}}$ \\
\hline 1,041 & 1 & $10,63^{\mathrm{ab}}$ & $16,81^{\mathrm{a}}$ & $13,72^{\mathrm{a}}$ & $0,653^{\mathrm{a}}$ \\
\hline 1,041 & 2 & $19,49^{\mathrm{ab}}$ & $24,65^{\mathrm{a}}$ & $22,07^{\mathrm{a}}$ & $0,602^{\mathrm{a}}$ \\
\hline 1,041 & 3 & $36,21^{\mathrm{ab}}$ & $40,68^{\mathrm{a}}$ & $38,45^{\mathrm{a}}$ & $0,480^{\mathrm{a}}$ \\
\hline 1,072 & 1 & $7,74^{\mathrm{ab}}$ & $14,57^{\mathrm{a}}$ & $11,16^{\mathrm{a}}$ & $0,646^{\mathrm{a}}$ \\
\hline 1,072 & 2 & $19,56^{\mathrm{ab}}$ & $25,09^{\mathrm{a}}$ & $22,33^{\mathrm{a}}$ & $0,586^{\mathrm{a}}$ \\
\hline 1,072 & 3 & $33,90^{\mathrm{ab}}$ & $37,45^{\mathrm{a}}$ & $35,68^{\mathrm{a}}$ & $0,493^{\mathrm{a}}$ \\
\hline 1,102 & 1 & $8,94^{\mathrm{b}}$ & $15,45^{\mathrm{a}}$ & $12,20^{\mathrm{a}}$ & $0,643^{\mathrm{a}}$ \\
\hline 1,102 & 2 & $17,19^{b}$ & $23,36^{\mathrm{a}}$ & $20,28^{\mathrm{a}}$ & $0,597^{\mathrm{a}}$ \\
\hline 1,102 & 3 & $32,22^{\mathrm{b}}$ & $37,48^{\mathrm{a}}$ & $34,85^{\mathrm{a}}$ & $0,496^{\mathrm{a}}$ \\
\hline
\end{tabular}

Médias com mesmo índice comparam os valores nas colunas não diferem significativamente em nível de $5 \%$ de probabilidade pelo teste de Duncan.

Tabela 3 - Patinamento dos pneus radiais traseiros, dianteiros, médio e eficiência de tração em função do solo solto, relação cinemática e cargas na barra de tração.

\begin{tabular}{|c|c|c|c|c|c|}
\hline \multirow{2}{*}{$\begin{array}{c}\text { Relação } \\
\text { Cinemática } \\
\text { (Kv) }\end{array}$} & \multirow[b]{2}{*}{ Carga } & \multicolumn{3}{|c|}{ Patinamentos } & \multirow{2}{*}{$\begin{array}{c}\text { Eficiência } \\
\text { de Tração } \\
(\%)\end{array}$} \\
\hline & & $\begin{array}{c}\text { Traseiro } \\
(\%)\end{array}$ & $\begin{array}{c}\text { Dianteiro } \\
(\%)\end{array}$ & $\begin{array}{c}\text { Médio } \\
(\%)\end{array}$ & \\
\hline 0,962 & 1 & $7,25^{\mathrm{ab}}$ & $14,00^{\mathrm{a}}$ & $10,63^{\mathrm{a}}$ & $0,610^{\mathrm{a}}$ \\
\hline 0,962 & 2 & $14,03^{\mathrm{ab}}$ & $20,22^{\mathrm{a}}$ & $17,13^{\mathrm{a}}$ & $0,593^{\mathrm{a}}$ \\
\hline 0,962 & 3 & $42,03^{\mathrm{ab}}$ & $47,10^{\mathrm{a}}$ & $44,57^{\mathrm{a}}$ & $0,416^{\mathrm{a}}$ \\
\hline 0,986 & 1 & $9,62^{\mathrm{a}}$ & $15,02^{\mathrm{a}}$ & $12,32^{\mathrm{a}}$ & $0,579^{\mathrm{a}}$ \\
\hline 0,986 & 2 & $17,12^{\mathrm{a}}$ & $23,45^{\mathrm{a}}$ & $20,29^{\mathrm{a}}$ & $0,580^{\mathrm{a}}$ \\
\hline 0,986 & 3 & $40,93^{\mathrm{a}}$ & $47,00^{\mathrm{a}}$ & $43,97^{\mathrm{a}}$ & $0,424^{\mathrm{a}}$ \\
\hline 1,009 & 1 & $7,73^{\mathrm{a}}$ & $14,11^{\mathrm{a}}$ & $10,92^{\mathrm{a}}$ & $0,598^{\mathrm{a}}$ \\
\hline 1,009 & 2 & $18,18^{\mathrm{a}}$ & $22,10^{\mathrm{a}}$ & $20,14^{\mathrm{a}}$ & $0,572^{\mathrm{a}}$ \\
\hline 1,009 & 3 & $42,44^{\mathrm{a}}$ & $46,72^{\mathrm{a}}$ & $44,58^{\mathrm{a}}$ & $0,410^{\mathrm{a}}$ \\
\hline 1,041 & 1 & $6,60^{\mathrm{ab}}$ & $13,05^{\mathrm{a}}$ & $9,83^{\mathrm{a}}$ & $0,603^{\mathrm{a}}$ \\
\hline 1,041 & 2 & $15,16^{\mathrm{ab}}$ & $20,67^{\mathrm{a}}$ & $17,92^{\mathrm{a}}$ & $0,578^{\mathrm{a}}$ \\
\hline 1,041 & 3 & $41,31^{\mathrm{ab}}$ & $44,95^{\mathrm{a}}$ & $43,13^{\mathrm{a}}$ & $0,422^{\mathrm{a}}$ \\
\hline 1,072 & 1 & $5,71^{\mathrm{ab}}$ & $11,44^{\mathrm{a}}$ & $8,58^{\mathrm{a}}$ & $0,608^{\mathrm{a}}$ \\
\hline 1,072 & 2 & $11,13^{\mathrm{ab}}$ & $16,98^{\mathrm{a}}$ & $14,06^{\mathrm{a}}$ & $0,606^{\mathrm{a}}$ \\
\hline 1,072 & 3 & $41,13^{\mathrm{ab}}$ & $53,21^{\mathrm{a}}$ & $47,17^{\mathrm{a}}$ & $0,393^{\mathrm{a}}$ \\
\hline 1,102 & 1 & $5,46^{\mathrm{b}}$ & $11,51^{\mathrm{a}}$ & $8,49^{\mathrm{a}}$ & $0,580^{\mathrm{a}}$ \\
\hline 1,102 & 2 & $9,75^{\mathrm{b}}$ & $15,31^{\mathrm{a}}$ & $12,53^{\mathrm{a}}$ & $0,601^{\mathrm{a}}$ \\
\hline 1,102 & 3 & $34,15^{\mathrm{b}}$ & $41,19^{\mathrm{a}}$ & $37,67^{\mathrm{a}}$ & $0,455^{\mathrm{a}}$ \\
\hline
\end{tabular}

Médias com mesmo índice comparam os valores nas colunas não diferem significativamente em nível de $5 \%$ de probabilidade pelo teste de Duncan. 


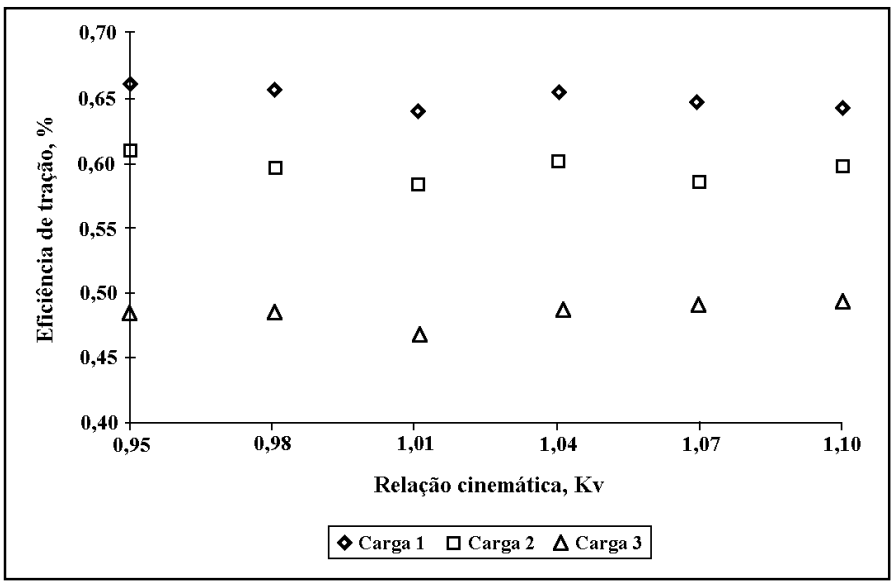

Figura 1 - Eficiências de tração do trator MF 620 com pneus radiais em solo firme, em função das relações cinemáticas estáticas.

e patinamentos médios entre os dois eixos não foram diferentes, em função das relações cinemáticas, ou seja, a variação dos Kvs não influenciou esses patinamentos. Os patinamentos dianteiros foram sempre maiores do que os patinamentos traseiros, mesmo com relações cinemáticas de atraso.

Quanto à eficiência de tração no solo firme, observa-se pela tabela 3 que não houve diferença entre as eficiências de tração, através da variação das relações cinemáticas entre os eixos.

Na figura 2, é apresentada a influência da relação cinemática na eficiência de tração em função das cargas impostas à barra de tração. Observa-se que as eficiências de tração estão bastante próximas, quando utilizadas as cargas 1 e 2 . Com a carga 3 , as eficiências de tração são reduzidas. Na carga 1 e na carga 2, a eficiência de tração tende a uma estabilização e indica que as maiores eficiências de tração

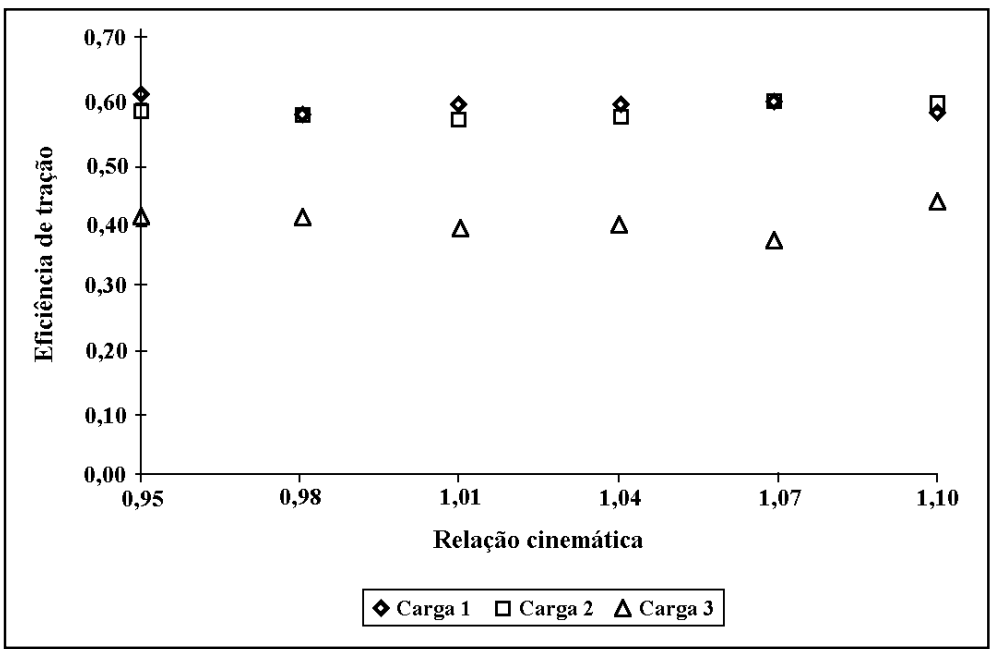

Figura 2 - Eficiências de tração do trator MF 620 com pneus radiais em solo solto, em função das relações cinemáticas estáticas. foram conseguidas com a relações cinemáticas de 1,072. Para a carga 3, observa-se que também ocorre uma estabilização das eficiências de tração, obtendo uma máxima eficiência com relações cinemáticas entre 1,041 e 1,102 , com uma pequena queda da eficiência na relação 1,072 .

\section{CONCLUSÕES}

A eficiência de tração não é influenciada pelas relações cinemáticas estáticas; para a condição de solo firme. As maiores eficiências de tração são obtidas com relações cinemáticas de 0,986 para cargas entre $21,2 \mathrm{kN}$ a $25,5 \mathrm{kN}$, relação cinemática de 1,041 para cargas de $25,7 \mathrm{kN}$ a $28,7 \mathrm{kN}$ e relações cinemáticas de 1,072 para cargas acima de $30 \mathrm{kN}$. Para a condição de tração em solo solto, as maiores eficiências de tração são obtidas com relações cinemáticas de 1,072 para cargas entre $13,8 \mathrm{kN}$ a $22,8 \mathrm{kN}$, e relações cinemáticas de 1,102 para cargas acima de $27,6 \mathrm{kN}$.

\section{REFERÊNCIAS BIBLIOGRÁFICAS}

CORRÊA, I. M., MAZIERO, J. V. G., MILAN, M. Tração dianteira auxiliar: Desempenho em função do pneu dianteiro. In: CONGRESSO BRASILEIRO DE ENGENHARIA Agrícola, 27, 1998, Poços de Caldas, MG. Anais... Poços de Caldas : Sociedade Brasileira de Engenharia Agrícola, 1998. 442p. p.154-156.

LINARES, P. Teoria de la tración de tractores agrícolas. Madrid. Madrid : Universidad Politécnica de Madrid, Escuela Técnica Superior de Inginieros Agrónomos, 1996, p.157.

MÁRQUEZ, L. Solotractor 90. Madrid : Laboreo, 1990. Parte III. Cap. 3.1: Adaptacion del tractor a la labor: p.128-145.

RACKHAM, D.H., BLIGHT, D.P. Fourwheel Drive Tractors - A Review. Journal Agric Eng Res, v.31, n.3, p.185-201, 1985.

SCHLOSSER, J.F. Influencia del avance cinemático de las ruedas delanteras motoras en la eficiencia en tracción de los tractores agricolas. Madrid: España, 1996. 234p. Tese (Doutorado em Agronomia) Departamento de Ingenieria Rural, Escuela Técnica Superior de Ingenieros Agrónomos, 1996.

Motores e tratores agrícolas. Santa Maria : Departamento de Engenharia Rural Universidade Federal de Santa Maria, RS. 1997 76p. (Caderno didático)

YANAI, K., CORRÊA, I. M., MAZIERO, J. V. G. Uso da tração dianteira auxiliar sob diferentes condiç̃es de lastragem e de pressão de inflação. In: CONGRESSO BRASILEIRO DE ENGENHARIA AGRÍCOLA, 1998, Poços de Caldas, MG. Anais ... Poços de Caldas, Sociedade Brasileira de Engenharia Agrícola, 1998. 442p. p.151-153. 